\title{
Interdependencies at work: \\ Constituting reflection, performance, dialogue and reward
}

\author{
Ray Smith and Stephen Billett \\ Griffith University, Australia
}

\begin{abstract}
This paper contributes to the ongoing debate about the relationship between the social and the individual as it is enacted in personal learning and the remaking of cultural practices through work. It discusses progress in a two-year study of the work, working lives and learning of twelve workers. They comprise four groups of three workers in an emergency service, gymnasium, restaurant and IT help desk work settings. The concept of relational interdependence between individual and social agency (Billett 2006), is used to understand how their participation, learning and remaking of cultural practices that comprises their work progresses. In identifying and elaborating bases of these interdependencies and their consequences for changes to individuals' cognitive experience and sense of self (i.e. learning), and the remaking of cultural practices (e.g. workplace practice), four linked and overlapping bases for understanding the processes of interdependencies emerge. These bases are: (i) reflection and review (i.e. reflection); (ii) performance roles (kinds of selves developed in the workplace); (iii) prospects for dialogue (i.e. opportunities for interpersonal interactions); and (iv) how conceptions of rewards and recognition are constructed. In different, but distinct, ways these four bases provide a means to elaborate interdependencies at work, thereby providing a platform to analyse processes of individual learning and the remaking of work practices and concepts throughout working life.
\end{abstract}

\section{Work, learning and the remaking of work}

There is enduring interest in the relationship between the social and the individual within the major disciplines of psychology (Rogoff, 1995; Scribner, 1997/1990; Valsiner, 1994), philosophy (Archer, 2000; Bhaskar, 1998) and sociology (Fenwick, 1998; Giddens, 1991) in understanding how people engage in social practices and learn through these experiences. Although different in emphasis, much of the deliberations in these disciplines are focused on the prominence of the contributions of either social structures or individual agency to cultural activity. Such debates address important issues associated with the origins of the knowledge required individually and societally required for participation in cultural activity, such as work. In addition, is the issue of whether and in what ways the individual or the social predominates in the formation (including its remaking and transformation) and learning of knowledge about and for work. These perspectives offer different responses to the question: do individuals bring about personal and cultural change, or is that change dependent upon social forms and structures (Ratner, 2000; Valsiner, 
2000)? Here, we propose that the answer is to be found in the dualities comprising relationship between the two. Workplaces provide a useful test bench to investigate and appraise this proposition because they are environments where the diversity of contributions necessary to the production of goods and services witness and manifest the enactments of knowledge that is socially-structured and individually-engaged.

In considering the dualities that comprise workers’ personal engagement in work and the transformations of learning and cultural practice that arise from this interactivity, this paper proposes some bases to identify how these dualities play out, can be illuminated and elaborated and understood further. These bases emerge from an ongoing investigation and analysis of groups of three workers each engaged in each of four distinct kinds of work and workplaces. Progressing from the conceptual positions outlined above, the paper is structured as follows. Firstly, the complex relational interdependence central to the dualities comprising the relationship between the social and the individual (or ontogenetic) contributions to individual learning and the remaking of practice are elaborated as the conceptual premises of this paper. Following this, the procedures adopted in the research project are described. Then, using an initial analysis of the data of just three workers, four bases for illuminating and elaborating both the relational interdependence and also the learning and remaking of practice are proposed. These comprise: (i) reflection and review (i.e. reflection); (ii) performance roles (kinds of selves developed in the workplace); (iii) prospects for dialogue (i.e. opportunities for interpersonal interactions); and (iv) how conceptions of rewards and recognition are constructed. Finally, some tentative propositions about the efficacy of these bases are advanced.

\section{Interdependencies at work}

Fundamental to the dualities involved in individuals' participation and learning through work are those comprising the affordances of the workplace, on the one hand, and individuals' engagement in the workplace, on the other (Billett, Barker and Hernon-Tinning 2004). Central to these dualities is the relational interdependence that arises between these affordances and individuals' engagement with them. This is because they interdependent in ways that are relational, rather than mutual or reciprocal. The social experience -- the social press -- does not and cannot exercise its suggestion comprehensively or unequivocally. As Newman, Griffin and Cole (1989) note, if the social suggestion was clear, unambiguous and potent, there would be little 
need to communicate because socially sourced and transmitted meaning would be understood unequivocally. It is proposed here that individuals have to engage in the process of remaking culture because they actively negotiate meaning and construe and construct what they experience, drawing upon their cognitive experience, which is in some ways unique to them (Billett 2003). So, neither the social suggestion nor individuals' agency alone is sufficient to understand learning and the remaking of the cultural practices that constitute work (Billett, Smith \& Barker 2005). Hence, both the social and personal are essential in terms of their contributions and how they mediate the agency of the other.

Indeed, the social suggestion or press comprising societal norms, practices and values, and their enactment in instances of social practices such as workplaces, is never complete or comprehensive enough to secure socialisation. Here, socialisation is defined as the unquestioned and comprehensive passage of knowledge from the social world to the individual and enculturation as the thorough and unequivocal induction into the particular practices that constitute that knowledge. The terms press and affordances are used here to indicate still other complexities such as the compelling and yet invitational qualities respectively of the cultural enactment of the social suggestion. Yet, as Berger and Luckman (1967) and others propose, the social suggestion is not projected in a way that is likely to lead to socialisation. This is because individuals will engage with the suggestion with greater or lesser reception, and greater or lesser fidelity in its appropriation. Because of limits in the social suggestion and the cultural practices into which they translate, individuals necessarily have to be agentic and active in the construction of meaning, if for no other reason than socially-sourced knowledge requires interpreting and construing to understand what is being suggested. Yet, even beyond simply attending to, engaging with and comprehending what is being suggested, individuals brings a possibly unique base of conceptions, procedures and values to their engagement with social forms and practices. This is because the processes and outcomes of engaging in work activities is more than the completion of tasks and interactions. For many, and perhaps most adults it is the means through which their identity is shaped and exercised (Noon \& Blyton, 1997; Pusey, 2003).

Therefore, and given the role that individuals' existing conceptions and processes play in the construal and construction of what they encounter, this process comprises a negotiated relationship arising through participation, with the process and 
outcomes of that engagement likely to be, in some ways, person dependent (Billett 2006). One way of understanding the learning that occurs through the processes of individuals' engagement with the social experience they encounter is to use concepts from the socio-cultural project, in particular the inter-psychological processes attributed to Vygotsky (1978): comprising those between the personal and social world. Links between self and work through inter-psychological processes of knowledge formation by individuals can be apprehended through understanding their engagement in ongoing and moment-by-moment individual learning or microgenetic development (Rogoff, 1990). Yet, on the cultural plane, another kind of development occurs. When individuals engage in work tasks and interactions, they are also actively participating in the remaking of those practices (Billett 2006).

These conceptual premises seem particularly salient for understanding the learning of the cultural practices that constitute paid work, through practice. Much of what needs to be learnt for vocational practice, has its origins in cultural practices and historical precedents (Scribner, 1985). Consequently, to access this knowledge with its important historical and cultural legacy requires engaging with the social world, because this knowledge does not arise from within the individual. Yet, when individuals engage with this knowledge and reconstruct it, in addition to their individual development, they are remaking these cultural practices at a particular point in time and under particular access to the social suggestion. Their enactment is not through faithful and mechanical enactments of the social suggestion that results in its reproduction. Instead, it is through individuals' engagement, construal of and construction of those practices, albeit mediated by the exercise of social and cultural norms and practices.

The exercise of personal agency, with its varying intensity and focus, is essential in transforming cultural practices because new cultural needs arise, such as those brought about by changing times or technologies. Wertsch (1998) distinguishes between compliant learning (i.e. mastery), which is superficial and may well be the product of forceful or compelling social suggestion of the kind which Valsiner (1998) identifies, and learning in which individuals engage willingly (i.e. appropriation) to a concurrence between what is experienced and individuals' values and beliefs. Richer or deeper kinds of learning requires effortful engagement buoyed by individuals’ interests and intentionality (Malle, Moses, \& Baldwin, 2001). Such learning is most likely to occur when individuals are engaged actively in the process of remaking 
practices. So, there is an inevitable interdependency between the agency of social world in projecting its suggestion and the agency of individuals in making sense of what is suggested to or afforded them by the particularities of their immediate work culture.

At the heart of individual agency is individuals’ personal epistemology, their idiosyncratic means of constructing personally relevant knowledge and meanings (Smith 2005) that is socially derived ontogentically, through a lifetime of social engagement, but mediates in personally unique ways what is subsequently experienced through their engagement in the affordances of the workplace. Fundamental to this mediation that constitutes the practice of personal epistemology is the active self-management of the diversity of personal and contextual influences that make up the work experience. That is, individual engagement, construal of and reconstruction of the cultural practices that constitute their work is not a haphazard affair but rather the evaluatively controlled management of the conduct and accomplishments of their work tasks and activities (Smith 2005). The personally agentic transformation of cultural practice, then, is not merely responsive or reactive to the social suggestion of the workplace. It is fundamental to it, relationally bound in the interdependencies that unite the individual worker and their work. However, it may seem accidental and or unexpected, in that intentional action and the purposes that guide it, however agentic in nature, cannot preclude the unintentional or the indeterminate (Gibbs 2001).

So, the kinds of participation, learning and the remaking of cultural practices that constitute work may sometimes seem incongruent with both the agency of the individual and that of the workplace. Therefore, it is important to acknowledge and account for the relational interdependencies that give rise to such dualities as individual and social contributions to participation in work, individuals' learning and the remaking of cultural practice and the processes and outcomes that manifest and sustain these complex relationships. These are the fields of inquiry that underpin the study reported here. Its preoccupation is the union of worker and change (both personal and cultural) through participation. The sociocultural project, with its origins in the research and theories of Vygotsky (1978) and others, seeks to explicate the nature of this union with its cognitive and cultural legacies through its analysis of the actions that constitute individuals' participation in the social world and particularly here, the world of paid employment. Mapping these participative activities and 
examining the nature of the relational interdependencies on which these activities are based is important work in advancing this understanding. The bases identified in this study and outlined below, offer a means to identify and elaborate some of the participative premises on which the relational interdependencies of participation in and transformation of work practices are established.

\section{Procedures}

To understand more clearly the relations that underpin the interdependencies at work and their consequences, the working lives of groups of three workers in each of four different kinds of occupations and workplaces was investigated. Selecting three workers across four workplaces was undertaken to gain insights into how affordances or invitational qualities of specific work cultures were exercised towards workers in the same workplace; how these workers then construed and constructed what was afforded them in the 'same' workplace setting; and how they engaged in their work and learned as result of these processes. Equally, this study aims to account for how different workplaces afforded opportunities for workers and workers, in turn, engaged with what was afforded them. Additionally, site selection was designed to gather data from sites with different purposes, organisational structures and patterns of employment. Grey (1994) indicated that institutional rules and practices have a significant impact upon the workplace participatory practices this study is seeking to identify and understand. Further, this study seeks to investigate different kinds of work, including some utilising technology and also teamwork; in order to gain insights in diverse work situations and workers whose employment standing and status were diverse.

The four workplaces selected for this study comprise: (i) a gymnasium; (ii) a restaurant; (iii) an Information Technology (IT) support section within a university; and (iv) a fire station. In each of these workplaces, three individuals agreed to participate. In the gymnasium -- Trim and Healthy -- they comprised a receptionist, a trainer and a manager. In the restaurant -- Platinum -- they comprised a chef, who is also a part owner, a waitress and a manager, who is also a part owner. In the IT support section, the participants comprised three helpdesk consultants, one of whom is a manager. In the fire station, the participants comprised a station manager, and two fire officers, one of whom is a specialist fire officer (i.e. ladder operator). 
The procedures adopted comprised a series of interviews that proceed over an 18-month period. The data gathering commenced with two lengthy semi-structured interviews, the first focusing primarily on the workplaces, their requirements for continuity and their norms and practices. The second interview focused primarily on the workers' history and personal preferences. These lengthy interviews were initial accounts of the affordances of the workplace and its practices: what constitutes their institutional facts (Searle, 1995) and also the bases for workers' participation in and learning for their work. Beyond these two initial interviews are a series of progress interviews occurring over a further year long period. These progress interviews are used to map changes in work practices, both personal and contextual, and the individuals' learning overtime. They are also used for the participants to comment on the initial analysis of the data from earlier interviews. The focus in this paper is on the first set of progress interviews for each of the 12 participants as they elaborate the bases of the relational interdependencies between workplace affordances and individual engagement in the four workplaces.

\section{Reflections, Performance role, Dialogue and Reward,}

Analysis of data from the first two interviews identified distinct bases through which these workplaces afford opportunities for engagement, learning and the remaking of the cultural practices across the four worksites (Billett, et al 2005). This included the relations between workplace affordances and how they are engaged with, construed and constructed by the individuals as exercised through their intentionality. However, the dualities identified are not simple. They are richly interconnected and intertwined. For example, the societal value and standing granted to fire fighters is something generated outside of their workplace but manifested in their practice and public execution of their work. The apparently contradictory mix of adherence to command and control protocols, on the one hand, and the opportunity afforded to have second forms of employment, on the other, are nuanced and relational bases for these workers to be highly integrated with their work practices and its culture. That is, beyond the sense of self that is afforded by the work, the conditions are also attractive, even within a highly top-down form of work organisation. In some ways, the work demands both this kind of engagement and the practice of command and control. Yet, clearly some contravene that requirement by not being wholly committed to their work, thereby raising questions about their competence to be relied upon during 
emergency work. By contrast, the intentions arising from the personal histories of the help desk workers are exercised in different ways within work that is hidden from many of those who benefit from it. Instead of public adulation and support (as in the case of the fire officers), some of these workers rely on electronic feedback from client surveys as a means to demonstrate their worth. Equally, the workers in the gymnasium and the restaurant are seeking quite different bases for their continuity and confirmation of their being effective through that work that shapes their intentions and agency. For some, this continuity comes from within the workplace (e.g. firefighters), but for others it comes from outside of it (part-time restaurant and gym workers).

These findings demonstrate how work and learning work are integrated in different ways and different purposes across these four workplaces. They emphasise the relational nature of the interdependencies between the social and individual contributions, yet articulates strongly how the confluence of the social and individual is exercised perhaps most strongly by individuals through their construal and construction (learning or practice of personal epistemology), and that the social practice is as much dependent on these individuals as individuals upon it. Hence, even from the perspective of the remaking of work practices, such as in those skills required for workplaces, the individual stands as an integral contributor and needs to be considered by both personal goals and pragmatic agendas currently associated with learning and learning throughout working life.

Following from those initial analyses, consideration of the data has turned to identify bases through which these dualities between affordances and engagements play out for these 12 workers. From an analysis of the progress interview data, factors associated with: (i) opportunities for reflection; (ii) construction of performance roles; (iii) opportunities and bases for dialogue; and (iv) constructions of reward emerged as ways to understand how these dualities play out. In the elaboration and illustration of these bases below, each is briefly described with an illustrative example of the data from one of the fire fighters.

\section{Reflection and review}

The constant demand for engaging with new knowledge and new practices that were required in these workplaces through changing customer and workplace requirements (e.g. restaurant, IT helpdesk), reshaping of the occupational practice (e.g. emergency 
services), technology (e.g. IT helpdesk) and work organisation (IT helpdesk and gymnasium), were reflective of changes to workplace requirements that are reported as being an increasingly general feature of contemporary work (OECD 2005). This demand for change can bring with it uncertainty, challenges to competence, new demands and stress. Consequently, opportunities provided by the workplace (i.e. affordances) for individuals to reflect upon these changes and respond to them, and how individuals elected to respond to these changes stand as a basis to understand and elaborate the relational interdependence between the individual and the social contributions.

The work and workplace of the fire fighters are rich with opportunity for reflection and review of both personal and contextual experience. The culture of care and concern that focuses on safety within the working operation of teams or 'crews' is integral to fire fighting work and promotes an awareness of self and others that in turn requires and develops the capacity for reflection and review. This work characteristic is supported by a number of primary workplace affordances that emphasise teamwork and the responsibilities and interdependencies by which it is defined. These include:

1) Shift allocation, that is, the placement and retention of the individual fire fighter within a team of usually four members for extended periods of time;

2) Shift rotation, the team working a shift of four $x 12$ hour days on and four off, two each by day and night, meaning that while on-station the team lives together - this is particularly important through the night shift where workloads are usually lighter and sleeping is permitted;

3) Designated roles and duties within the team, (eg, driver, pump operator, etc), that differentiate individuals and define working relationships within the team;

4) Regular in-shift team training exercises; and

5) Relatively large amounts of down-time that enable a high degree of interaction and personal communication around work and non-work related experiences. Essentially, these workplace affordances bring specific fire fighters together for the same related practices over relatively extended periods of time in the same place. To elaborate the relational nature of these workplace participatory practices, it is worth considering the example of Hugh.

Hugh has successfully completed the 13 week recruitment training and exam and is currently working his way through the self-paced training modules that will, over a three years period, lead to a diploma qualification necessary for continued 
employment in the fire service. Yet he also refers to other kinds of affordances. You learn by going to incidents, witnessing things and then talking to the boys afterwards. A big part of our job is a debrief among the boys because you don't see everything there's so much action going on that you only see your little bit. For Hugh, the immediacy of the hands-on experience at incidents is central to learning. This is linked to the need for a debrief after the incident that forms the third component in the learning chain -do, witness then talk. He makes a point of returning to the site after the incident if possible. This process allows you to work out what was actually there as opposed to what you perceived was there through all the smoke and confusion and the limited vision that is afforded by doing your little bit. Without this process I go away from the incident with a feeling that there's something missing, I didn't get the whole picture. Sometimes what you see afterwards doesn't relate to the picture your mind built up through all the smoke and limited visibility. For me, it finalises things and gives you something more to build on for next time. It's a personal learning process that makes me a better fire fighter.

In this way, the duality of the affordance of the work practice and worker's agency and intentionality in engaging in and reflecting upon work activities serves to illustrate the relational basis of this interdependency. Similar processes of reflection and review were discernable in the other workers.

\section{Performance roles}

Emphasising more a focus on the individual contributions were capacities to be seen to be and performing particular roles. This included specialist skills within work teams (e.g. IT helpdesk, emergency services), a training role given to a part-time worker and a marketing function taken by another (i.e. restaurant owner/manager), being self-employed individual for one worker and a more corporate management role for another (i.e. gymnasium) were identified as important performance roles that were being constructed in these four work settings. However, these roles were not uniformly welcomed. For instance, the part-time waitress was not seeking to expand her roles and responsibilities in the restaurant despite the manager's belief in her capacities. Instead, she wanted to merely secure remuneration from the restaurant that would support her through university. Also, this knowledge was constructed through negotiated relations between individual and workplace goals (IT helpdesk and gymnasium). In the IT helpdesk, although working in a shared space and on similar 
work, these workers' trajectories and preferences were quite distinct. Here, issues of self and the intentionality of individuals' participation and remaking practice come together. That is, the individual who takes upon himself or herself a particular performance role is seeking to exercise that role for particular intentional purposes. These individual purposes will be consistent or inconsistent by degree with those of the workplace. Moreover, the degree by which the role is welcomed and is successfully anointed by the workplace is linked to its meeting the workplace's trajectory and goals.

The emergency service work requires fire fighters to enact a number of roles, often simultaneously. For Hugh, this is a junior fire fighter - who holds the designated position of seat number 4 in the appliance (i.e. water pump vehicle) that takes crews to emergency incidents. In the multiple crewed station where he works, there is provision for only 2 junior fire fighters. However, Hugh relishes the role of a junior fire fighter. His commitment to this position includes the completion of a diploma within the allocated 3 years. Yet beyond this he is afforded on-going development in the workplace best characterised as an apprenticeship with all the learning experiences of that training model. Despite his position being recognised as junior and, therefore, inexperienced, Hugh brings a high level of experience to this position as a result of years of service as a fire fighter in Britain. So, Hugh does not personally identify as an inexperienced fire fighter, but as a transferee to another service that failed to recognise his experiences and expertise. He recounts stories of his 'inexperience' in the British fire fighting service and the learning by mistakes that resulted, eg, the axe through the plate glass window. Yet his 'inexperience' does not personally accord with his afforded role of junior fire fighter. He notes the level of support he is afforded in this role is different than that in Britain where inexperienced fire fighters are known and treated as the 'sprog'. There are some long serving boys here and I'm not treated as the sprog as such because that culture is fast fading and my experience is recognised by the boys here who may have been in longer here in Queensland than I have but I've seen a lot more fires than they have.

In this way, similarly, the duality of performance roles are elaborated in what roles and support for those roles are afforded and also how those roles are engaged with by individuals in different degree and for different kinds of purposes. Consequently, the capacities for and judgements about performance roles stands as a 
basis to understand the relational interdependence between personal and social factors that shape participating in and learning through work.

\section{Dialogue}

In addition to managing the change requirements of work, dialogue with others was identified as a basis for ongoing work and work practice, and the maintenance of performance roles. So, the process of, opportunities for and bases of dialogue were central to the performance of work, learning and remaking practice. Yet, this important process was, not surprisingly, afforded in different ways in different workplaces and the same workplace. Moreover, its engagement by individuals was not wholly a product of what the workplace afforded.

In Hugh's situation, the opportunity is afforded to engage in informal debriefs in the appliance when returning from incident and over a cup of tea on return to station. Formal debriefs and professional counselling are available after major incidents. However, he suggests "you cant beat the boys sitting down and having a good chat about what they saw, especially if you witnessed something not nice yourself-you can get it out of your system, talk about it - it just helps alleviate stress and that type of thing". He also notes the importance of "talking to other fireys to relate their experiences - that's a big part of the culture, sitting around with a cup of tea chatting about the job. They'll pass on their experiences". However, he also comments that often, these dialogues arise "through someone's failure to do something. Once recognised others chime in with their similar experiences and you learn something”. Yet, this dialogue is not only between co-workers, it is intentionally afforded by the workplace. Critical stress management policy requires officers to check fire fighters’ need for counselling after major incidents. Moreover, there are norms and conventions within the workplace about how these dialogues progress. Hugh refers to exercising some caution in conversations, especially with older more experienced fire fighters. He noted the need to approach the situation correctly. They are very willing to share that knowledge with you. If you go about it the wrong way, take them for granted, get too selective with what you're trying to learn, picky, they can shut down a bit but if you're receptive to everything they're saying to you, prepared to listen - sure you filter it with what's relevant to more modern times - they can impart some fantastic knowledge. So, there are relationshipbuilding skills involved here that reflect the negotiations between individuals and the 
social practice of the work place. Yet despite the availability of opportunities the team work provides, communication and particularly conversation is not guaranteed here. Away from the immediacy of group tasks and informal chat, some will want to read, to exercise in the gym, watch television or simply sleep without interruption from well meaning colleagues So, learning and gaining access to "the important stuff that's outside the manuals, the knowledge of experience" that resides with individuals, requires personal effort and sensitivity to the needs of others.

Therefore, both ontogenetic and situational contributions serve to underpin the bases of dialogue through which participation, learning and remaking of work practice occur. Consequently, dialogue stands as an important premise for understanding the processes of the relational interdependence between social and personal factors.

\section{Rewards}

Rewards have a dual form; both those central to individuals' goals and those that workplaces are directed to achieving. Securing rewards was identified as exemplifying key purposes towards which individuals were driven in their work and their remaking of their work practices. Yet, their actions reflected the duality of participating in work, learning and remaking practice. The workplaces afforded different kinds of rewards, in different ways and for different purposes. However, individuals also constructed rewards that were separate from (although not necessarily unaligned to) the requirements of the workplace, on the bases of their own needs and trajectories. Learning rewards are also afforded by the workplace setting. After major incidents, opportunities are provided to build context and a sense of personal purpose and performance in team response through debrief and or return to site - Its nice to go back in and check how accurate your brain picture was so next time you'll know what something is by the ways it feels - it fills in the blanks - gives you an understanding to fall back on when you next have very restricted visibility. Without this process I go away from the incident with a feeling that something is missing, I didn't get the whole picture. Learning rewards for Hugh are when prior learning serves to solve an immediate workplace problem. Yet another type of reward is afforded when the culture of care and safety extends to protection of crew by each other from the potential threat of other fire fighters with a reputation for laxity. Being warned is a reward of acceptance, inclusion. 
There are other kinds of rewards provided by this workplace that permit continuity of employment, long non-work periods between shifts and opportunities to progress through the organisation. Moreover, as the physical work required in firefighting and emergency service work is less easier to do later in working life, there are opportunities and positions available that permit fire fighters to retain their identity as fire fighters, yet to work in roles that are commensurate with their physical capacities. Again, both the rewards afforded by the workplace and those that are central to individuals' identity as a worker (e.g. fire fighter) are exercised in a way that is interdependent. The prospect for both is entwined and interwoven with the other. Yet, rewards reflects both personal and institutional goals and stand to assist understanding what directs both individuals' engagement and workplace affordances. In this way, rewards capture the goals for both workplace and personal trajectories, and permits a consideration of the degree by which there is consonance between these trajectories. Hence, they richly inform the relational interdependence between the personal and social.

\section{Prospects for further elaborations}

Here, four bases for understanding and appraising the relational interdependence between the social and individual contributions to participation, learning and remaking of cultural practice have been identified and presented. An instance based largely on data from just one subject has been used to illuminate these relations in this section. The data from other fire fighters in this same workplace (e.g. one who works from home while tending to his sick wife, another who is a senior officer) offer different sets of relationships that serve to shape their kind of participation, learning and remaking of practice. Across these other firefighters and similarly for workers in the other three workplaces, these bases are also played out in different ways premised on quite different relations. Further, across these sites, there are quite different institutional arrangements for participation and learning (Billett et al 2005). It is intended to explore further these workers' experiences through the bases for understanding the relational interdependence as outlined above. It is clear that quite different and distinct opportunities for reflection, dialogue, rewards and performance roles are afforded and exercised across these workplaces.

Moreover, the initial data suggests there are stronger distinctions and differences within these workers' goals, their intentions for participating in work and 
how they exercise their interest and agency. Of particular interest are the ways in which the bases outlined interrelate to illuminate the social process of personally negotiating the relational nature of the interdependencies that unite workers and the changes that constitute their work, learning and cultural practice. For example, and by way of returning to the initial conceptual premises of this paper, each of the workplaces afforded workers formally organised opportunities to review and respond to the changes characterising their working and learning practices. In the case of the fire service, these staff meetings were more than the cake and coffee conversations that were the norm for the IT workers. Following major emergency incidents, they were formal debriefing sessions with the specific learning objective of reviewing for improved performance at future incidents. Under such circumstances, and by contrast with the more informal gatherings discussed earlier, the interrelationships of the four bases outlined here indicate clear distinctions between the kinds of roles, practices and perceptions individual workers undertake and draw upon. That is, the dualities of workplace affordance and worker engagement reveal ways in which workers personally negotiate their circumstances and contributions to the interdependencies from which personal and cultural change derives.

Further, of particular interest is how the bases identified may be simultaneously sourced within these changes, that is, as both emergent outcomes of and processes for change. Hugh is clearly not an 'inexperienced' fire fighter and highly values the workplace affordances that enable him, through dialogue, to access the wealth of experience his colleagues carry. However, he likewise brings a wealth of experience to these interactions and therefore stands as potentially influential in others of the changes this learning represents for him. As he negotiates his participation relative to the affordances of colleagues, he is constantly in the process of evaluating how his colleagues value his contributions relative to his own understandings of his performance and contributions to the team effort that is his work. The bases identified offer some way into these complex dualities that unite personal engagement and the kinds of changes that evidence learning and the remaking of cultural practice as both processes for and outcomes of participation in work. Given the diversity of relations that have been identified in each workplace, and the kinds of individual intentions that are enacted in these four workplaces in participation in work, and hence, individuals' learning and their remaking of practice, the study has begun to illuminate the diverse premises for learning through and for 
work and throughout working life. Clearly, it is issues of self, personal goals and needs that direct individuals in these activities. These may be remote from what employers and governments intend through their efforts to secure economic reform and development through their promotion of lifelong learning.

Acknowledgements. The authors wish to acknowledge the support provide by the Australian Research Council to conduct this research, the Australian Postgraduate Award that, in addition, supports the work of Ray Smith and also the time, commitment and other contributions of the participants and their workplaces. Also, thank you to the editor and reviewer for helpful comments that assisted in the improvement of the paper.

\section{References}

Archer, M. S. (2000). Being Human: the problem of agency. Cambridge: Cambridge University Press.

Berger, P. L., \& Luckman, T. (1967). The Social Construction of Reality. Harmondsworth, Middlesex: Penguin Books.

Bhaskar, R. (1998). The possibility of naturalism. London: Routledge.

Billett, S. (in press 2006) Relational interdependence between social and individual agency in work and working life. Mind, Culture and Activity (13) 1

Billett S (2003) Sociogeneses, activity and ontogeny. Culture and Psychology, 9 (2) 133-169

Billett, S., Smith, R \& Barker, M. (2005) Understanding work, learning and the remaking of cultural practices. Studies in Continuing Education 27 (3) 219-237.

Billett, S., Barker, M., \& Hernon-Tinning, B. (2004). Participatory practices at work. Pedagogy, Culture and Society, 12(2), 233-257.

Fenwick, T. (1998). Women's Development of Self in the Workplace. International Journal of Lifelong Learning, 17(3), 199-217.

Fenwick, T. (2002). Lady, Inc.: women learning, negotiating subjectivity in entrepeneurial discourses. International Journal of Lifelong Education, 21(2), 162-177.

Gibbs, R. (2001) Intentions as emergent products of social interactions. In In B. F. Malle, L. J. Moses \& D. A. Baldwin (Eds.), Intentions and Intentionality: Foundations of Social Cognition (pp. 105-122). Cambridge, Massachusetts: The MIT Press.

Giddens, A. (1991). Modernity and self-identity : Self and Society in the Late Modern Age. Stanford: Stanford University Press.

Grey, C. (1994). Career as a project of the self and labour process discipline. Sociology, 28(2), 479-497.

Malle, B. F., Moses, L. J., \& Baldwin, D. A. (2001). Introduction: The Significance of Intentionality. In B. F. Malle, L. J. Moses \& D. A. Baldwin (Eds.), Intentions and Intentionality: Foundations of Social Cognition (pp. 1-26). Cambridge, Massachusetts: The MIT Press.

Newman, D., Griffin, P., \& Cole, M. (1989). The construction zone: Working for cognitive change in schools. Cambridge, UK: Cambridge University Press.

Noon, M., \& Blyton, P. (1997). The realities of work. Basingstoke, Hants: Macmillan.

OECD (2005). Trade and structural adjustment: embracing globalisation. In Industry, Services and Trade, 2005(28), 1-338.

Pusey, M. (2003). The Experience of Middle Australia. Cambridge, UK: Cambridge University Press.

Ratner, C. (2000). Agency and culture. Journal for the Theory of Social Behaviour, 30(413434).

Rogoff, B. (1990). Apprenticeship in thinking - cognitive development in social context. New York: Oxford University Press.

Rogoff, B. (1995). Observing sociocultural activity on three planes: Participatory appropriation, guided participation, apprenticeship. In J. W. Wertsch, A. Alvarez \& P. 
del Rio (Eds.), Sociocultural studies of mind (pp. 139-164). Cambridge, UK:

Cambridge University Press.

Scribner, S. (1985). Vygostky's use of history. In J. V. Wertsch (Ed.), Culture, communication and cognition: Vygotskian perspectives (pp. 119-145). Cambridge, UK: Cambridge University Press.

Scribner, S. (1997/1990). A sociocultural approach to the study of mind. In E. Tobah, R.J. Falmagne, M.B. Parlee, L.M. Martin \& e. A.S. Kapelman (Eds.), Mind and Social Practice: Selected Writings of Sylvia Scribner. (pp. 266-280.). Cambridge, UK: Cambridge University Press.

Searle, J. R. (1995). The Construction of Social Reality. London: Penguin.

Smith, R. (2005) 'Epistemological agency and the new employee', Australian Journal of Adult Learning, 45(1):29-46.

Somerville, M. (2002). Changing Masculine Work Cultures. Paper presented at the Envisioning practice -- Implementing change, Gold Coast.

Valsiner, J. (1994). Bi-directional cultural transmission and constructive sociogenesis. In W. d. Graaf \& R. Maier (Eds.), Sociogenesis Re-examined (pp. 101-134). New York: Springer.

Valsiner, J. (1998). The Guided Mind: A Sociogenetic Approach to Personality. Cambridge, Mass: Harvard University Press.

Valsiner, J. (2000). Culture and Human Development. London: Sage Publications.

Vygotsky, L. S. (1978). Mind in society - the development of higher psychological processes. Cambridge, Mass: Harvard University Press.

Wertsch, J. V. (1998). Mind as Action. New York: Oxford University Press. 